\title{
Benefícios económicos e ambientais inerentes ao uso de materiais estruturais naturais em habitações unifamiliares
}

\author{
Economical and environmental benefits related to the \\ use of natural materials in single-family house-buildings
}

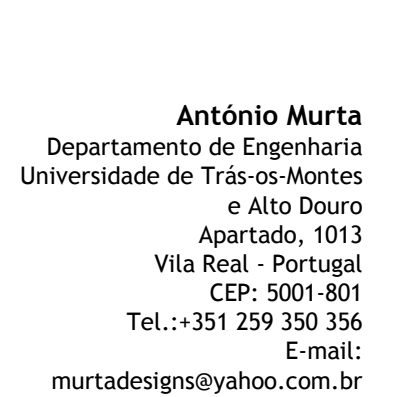

Humberto Varum Departamento de Engenharia

Universidade de Aveiro Campus Universitário de Santiago

Aveiro - Portugal

CEP: $3810-193$

Tel.: +351234370938

E-mail: hvarum@ua.pt

Jorge Pinto

Departamento de Engenharia Universidade de Trás-os-Montes

e Alto Douro

Tel.:+351259350 356

E-mail: tiago@utad.pt

Isabel Bentes

Departamento de Engenharia Universidade de Trás-os-Montes

e Alto Douro

Tel.:+351259350 356

E-mail: ibentes@utad.pt

Anabela Paiva

Departamento de Engenharia Universidade de Trás-os-Montes e Alto Douro

Tel.:+351 259350356

E-mail: apaiva@utad.pt

Luis Ramos

Departamento de Engenharia Universidade de Trás-os-Montes

e Alto Douro

Tel.:+351259350356

E-mail: Iramos@utad.pt

Recebido em 19/02/2010

Aceito em 07/08/2010

\section{António Murta \\ Humberto Varum \\ Jorge Pinto \\ Isabel Bentes \\ Anabela Paiva \\ Luis Ramos \\ Resumo}

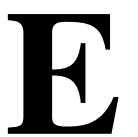

m Portugal, a indústria de construção habitacional tem recorrido essencialmente às estruturas de betão armado e de alvenaria de tijolo cerâmico. As estruturas metálicas, de madeira e de alvenaria de pedra têm tido expressão reduzida nesse setor da construção, e são muito pontuais as aplicações da terra crua como solução construtiva. Nesse contexto, pretende-se estudar soluções construtivas mais sustentáveis, recorrendo a materiais de construção naturais. Para o efeito, foram estudadas para uma habitação unifamiliar com tipologia típica três soluções estruturais: estrutura porticada de betão armado e lajes aligeiradas pré-fabricadas; solução estrutural à base de materiais naturais; e solução que contempla a reutilização de madeira proveniente de demolições habitacionais. As soluções estruturais com recurso a materiais naturais estudadas são definidas por paredes resistentes de bloco de terra comprimida (BTC) e estrutura de madeira nos pavimentos e cobertura. Essas soluções estruturais são comparadas em termos de custo, gastos energéticos e teor de emissão de $\mathrm{CO} 2$ associados à construção. Verificou-se que as soluções mais sustentáveis são muito mais vantajosas no que diz respeito a esses três aspectos.

Palavras-chave: Materiais e técnicas de construção. BTC. Energia incorporada. Emissão de $\mathrm{CO}_{2}$. Avaliação de sustentabilidade na construção.

\section{Abstract}

In Portugal, the house building industry has mostly adopted reinforced concrete framed structures and clay brick-wall. The use of other structural materials, such as steel, timber, and stone-wall is still rather limited in this sector. Moreover, the use of earth-based construction techniques and solutions still remains limited to individual cases. Within this context, this paper describes an investigation on sustainable structural solutions for a typical single family house using natural materials. Three different structural solutions were compared, namely a reinforced concrete frame solution, a solution based on CEB (compressed raw earth blocks) masonry and timber structures for floors and roof, and a solution based on reusable materials, such as timber originated from the demolition of buildings. These alternative structural solutions were compared in terms of building costs, energy consumption and $\mathrm{CO}_{2}$ emissions. The study confirmed that the sustainable solutions had most advantages, particularly with regards to the environment.

Keywords: Building materials and techniques. CEB. Incorporated energy. CO2 emissions. Sustainability evaluation in construction. 


\section{Introdução}

A terra é um material utilizado para a construção de habitações em todo o mundo (MINKE, 2005). Como tal, uma vasta percentagem da população usufrui desse tipo de edifício. De acordo com Minke (2005), cerca de metade da população mundial, aproximadamente 3 biliões de pessoas em seis continentes, habita ou trabalha em edifícios construídos em terra (MINKE, 2005). Já Pinto et al. (2008) defendem que em alguns países com menores recursos económicos a construção em terra poderá representar mais de metade da totalidade das construções existentes. De acordo com as estatísticas da United Nations Centre for Human Settlements (UNCHS), 40\% da população mundial vive em habitações de terra (AUROVILLE EARTH INSTITUTE, 2010). Embora não exista coincidência nos números defendidos pelos vários autores, é muito expressivo o número de pessoas que usufrui desse tipo de edifícios.

Podemos, no entanto, afirmar que a construção de terra surge em muitas construções classificadas como património da Humanidade e que é elucidativo da diversidade cultural dos povos e demonstrativo da sua versatilidade em termos de aplicação (CORTÉS, 2009). Além disso, de acordo com a United Nations Educational, Scientific and Cultural Organization (UNESCO), $17 \%$ do património cultural mundial é construído em terra (AUROVILLE EARTH INSTITUTE, 2010).

Os materiais e elementos de construção à base de terra são mais sustentáveis, por um lado, porque a terra é natural, reciclável e abundante em qualquer local e, por outro lado, porque as técnicas utilizadas para o fabrico desses materiais e elementos construtivos são normalmente simples, requerem pequena quantidade de energia e emitem uma quantidade inexpressiva de gases nocivos para a atmosfera.

A obtenção dos materiais correntes na construção atual em Portugal requer bastante energia durante as várias fases (extração, transporte, transformação, aplicação, demolição e eliminação ou reciclagem), associada à libertação de gases poluentes para a atmosfera em quantidades significativas.

Com este trabalho estuda-se a viabilidade de soluções construtivas mais sustentáveis, aliando a aplicação de materiais naturais, de fácil obtenção perto do local de construção, a métodos construtivos que requerem pouca energia na sua aplicação e, implicitamente, baixo teor de emissão de gases poluentes para a atmosfera. Nesse sentido, estuda-se a aplicação de blocos de terra comprimida para a construção de habitações unifamiliares correntes.

Definiu-se uma tipologia corrente de habitação unifamiliar para a qual foram estudadas paralelamente três soluções estruturais - uma "solução tradicional" e duas soluções mais sustentáveis. A solução tradicional é a do tipo pilar-viga de betão armado com lajes aligeiradas de vigotas pré-esforçadas nos pavimentos e também lajes maciças em certos elementos como varandas e escadas, e alvenarias de tijolo cerâmico nas paredes divisórias e exteriores. A primeira solução mais sustentável (solução sustentável I) é definida por paredes resistentes de blocos de terra comprimida (BTC) e pavimentos, cobertura e escadas de madeira. A segunda solução mais sustentável (solução sustentável II) é bastante semelhante à primeira, diferindo apenas no fato de se reutilizar madeira proveniente da demolição de edifícios.

Para cada uma dessas três soluções estruturais foi quantificado o consumo energético, a emissão de gases poluentes para a atmosfera e o gasto energético. Por comparação conclui-se que as soluções estruturais mais sustentáveis estudadas são inequivocamente mais vantajosas em termos económicos e ambientais. Adicionalmente, também se observou que a reutilização de materiais de construção, a madeira neste caso, acentua as vantagens referidas anteriormente.

Numa reflexão final, são indicadas soluções que permitem estimular a aplicação de soluções estruturais mais sustentáveis em edifícios de habitação unifamiliar mesmo de habitação.

\section{Descrição do edifício estudado}

O edifício estudado é idealizado como sendo localizado na cidade da Figueira da Foz. A implantação do edifício é assumida em zona urbana, com envolvente de edifícios de pequeno porte, a uma altitude de $100 \mathrm{~m}$.

Neste estudo a tipologia assumida é considerada como de grande procura no mercado português (cerca de 57\%) (PORTUGAL, 1998) para o alojamento familiar clássico. Esse tipo de habitação é adequado a um agregado familiar composto de 3 a 4 pessoas.

O edifício de habitação unifamiliar é muito simples em termos de arquitetura, volumetria e distribuição de espaços (Figuras 1 a 4). Os espaços foram 
distribuídos por dois pisos, dedicando a utilização do piso térreo (piso 0) essencialmente a funções do tipo social e a utilização do piso superior (piso 1) essencialmente ao uso privado. As áreas brutas de construção dos pisos 0 e 1 são de $285,00 \mathrm{~m}^{2}$ e $106,50 \mathrm{~m}^{2}$ respectivamente.

O piso 0 é formado por uma sala de estar, uma sala de jantar, uma cozinha, uma zona de arrumos, instalações sanitárias, hall de entrada e corredor, existindo ainda um alpendre que se desenvolve ao longo de duas fachadas do edifício. No piso 1 existem dois quartos (um deles com vestiário privativo), um escritório, instalações sanitárias, hall de distribuição e uma varanda.

A ligação entre pisos é efetuada por uma escada que liga o hall de entrada no piso 0 ao hall de distribuição no piso 1. A cobertura do alpendre é inclinada de apenas uma água, com revestimento em telha cerâmica, e a cobertura do piso 1 é horizontal (terraço não acessível), com revestimento de terra vegetal.

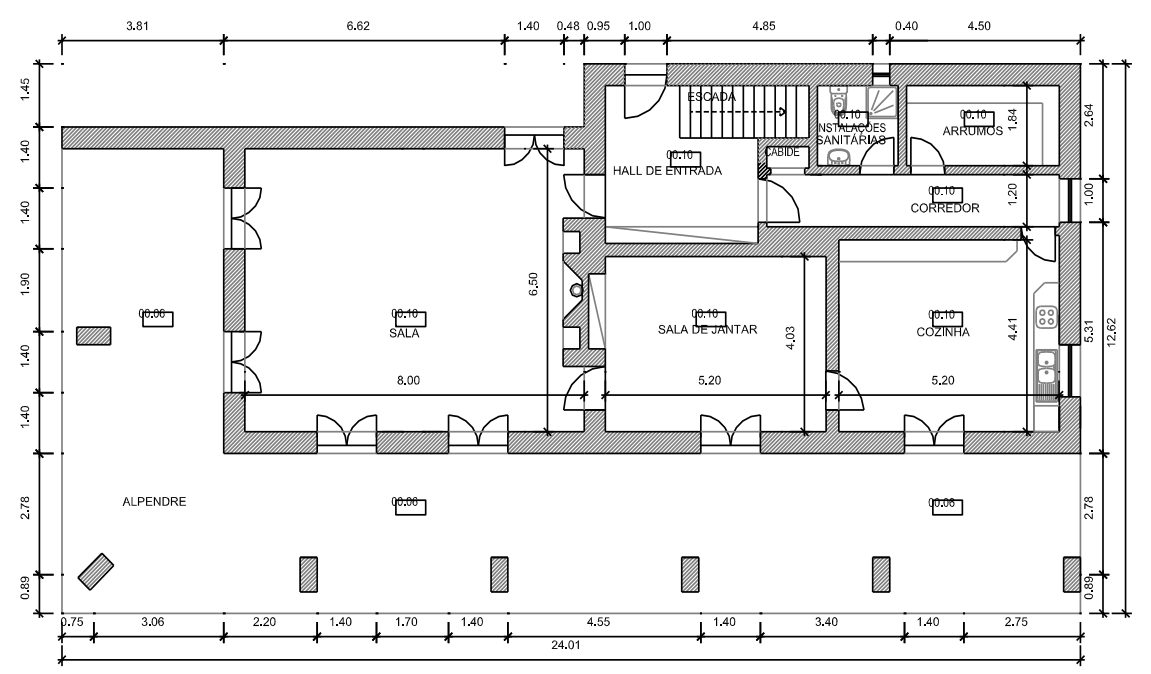

Figura 1 - Arquitetura - Planta do piso 0

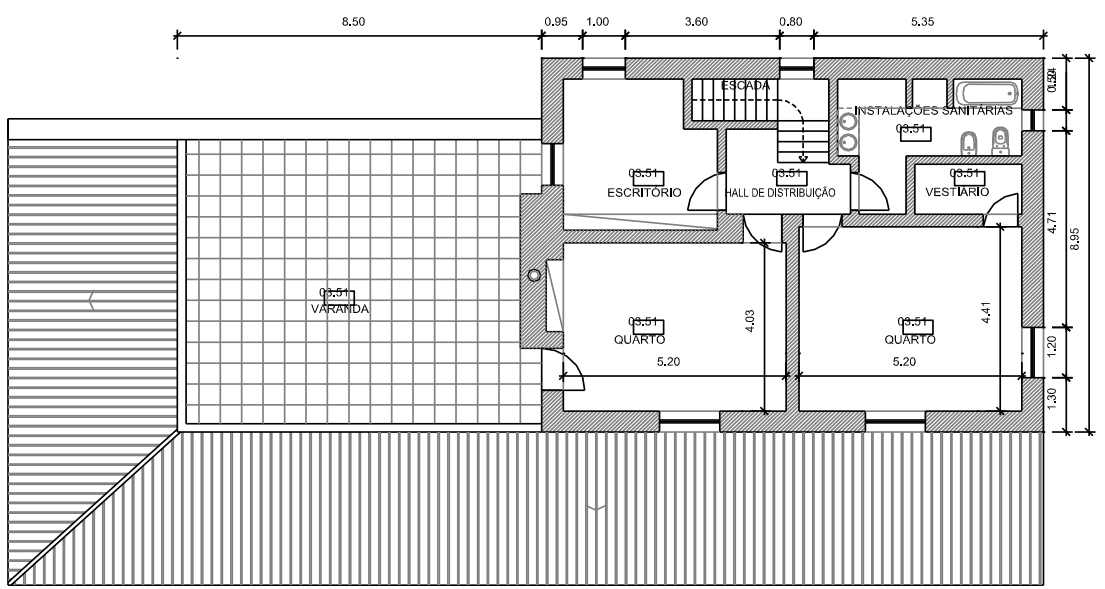

Figura 2 - Arquitetura - Planta do piso 1 


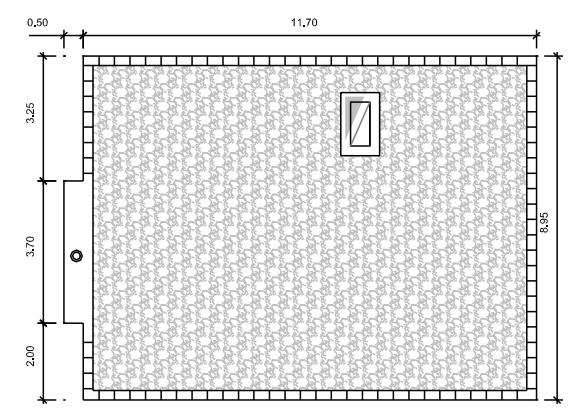

Figura 3 - Arquitetura - Planta da cobertura

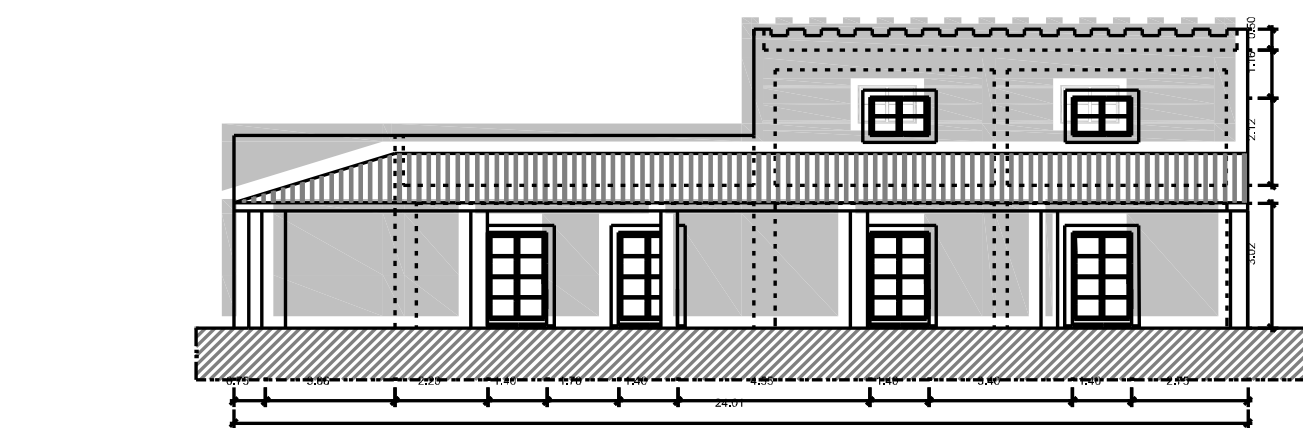

Figura 4 - Arquitetura - Alçado oeste

\section{Análise estrutural das 3 soluções estudadas}

Para o dimensionamento da solução estrutural tradicional em betão armado, utilizaram-se programas de cálculo automático comerciais. O programa de cálculo utilizado permite efetuar uma análise dinâmica modal, com avaliação prévia dos vários modos de vibração e determinação da resposta da estrutura considerando a ação sísmica através de espectros de resposta. A quantificação da ação do vento é feita de modo automático pelo programa de cálculo, após indicação da zona e da rugosidade associada ao local de implantação do edifício, bem como das dimensões do edifício em planta. Foram elaboradas algumas folhas de cálculo em Excel para a verificação e análise dos resultados obtidos através do programa de cálculo automático. Essas folhas de cálculo foram também utilizadas para o dimensionamento de alguns elementos estruturais isolados, tais como vãos de escada.

Para o estudo das soluções mais sustentáveis desenvolveram-se folhas de cálculo em Excel para o dimensionamento e verificação da segurança dos elementos de alvenarias de BTC, com base no disposto na regulamentação Eurocódigo 6
(EUROPEAN COMMITTEE FOR STANDARDIZATION, 2005a) e Eurocódigo 8 (EUROPEAN COMMITTEE FOR STANDARDIZATION, 2005b). Para o dimensionamento dos elementos estruturais de madeira (pavimentos, cobertura e escadas) recorreu-se ao disposto no Eurocódigo 5 (EUROPEAN COMMITTEE FOR STANDARDIZATION, 2004) e também se elaboraram folhas de cálculo específicas, em Excel, para sistematização dos cálculos.

\section{Solução tradicional}

Para o edifício em estudo, e de acordo com REBAP (PORTUGAL, 1983), considerou-se um betão do tipo $\mathrm{C} 25 / 30$ em todos os elementos estruturais (sapatas, pilares, vigas, escadas e lajes). $\mathrm{O}$ aço considerado nas armaduras correntes foi $\mathrm{o}$ S400NR, e foi usado o aço S500EL na malha eletrossoldada da camada de compressão das lajes aligeiradas de acordo com REBAP (PORTUGAL, 1983). As paredes exteriores serão duplas em alvenaria de tijolo cerâmico e as paredes interiores serão constituídas por panos simples de alvenaria de tijolo cerâmico, ambas argamassadas nas juntas e rebocadas. 
Para a análise estrutural do edifício foi necessário quantificar inicialmente as ações a considerar e estimar os seus efeitos nos elementos estruturais. A ação permanente considerada corresponde ao peso próprio dos materiais (estruturais e não estruturais). As ações variáveis contempladas foram a sobrecarga, o vento e o sismo. Para o peso próprio dos elementos estruturais foram considerados os pesos específicos de referência de $78,50 \mathrm{kN} / \mathrm{m}^{3}$ para o aço e de $25,00 \mathrm{kN} / \mathrm{m}^{3}$ para os elementos de betão armado. Para o revestimento dos pisos e da cobertura considerou-se $2,00 \mathrm{kN} / \mathrm{m}^{2}$ e $3,00 \mathrm{kN} / \mathrm{m}^{2}$ respectivamente.

Para as sobrecargas de utilização, e segundo o descrito em RSA (PORTUGAL, 1985), considerou-se $2,00 \mathrm{kN} / \mathrm{m}^{2}$ para as zonas habitáveis, $1,00 \mathrm{kN} / \mathrm{m}^{2}$ para os terraços não acessíveis e 3,00 $\mathrm{kN} / \mathrm{m}^{2}$ para zonas de acesso. Para a ação do vento, e segundo RSA (PORTUGAL, 1985), considerouse que o edifício está localizado na zona B, com uma rugosidade do tipo II. Para a ação sísmica, e segundo RSA (PORTUGAL, 1985), considerou-se a zona sísmica $\mathrm{C}$ (coeficiente de sismicidade de 0,50), terreno do tipo II, coeficiente de amortecimento de $5 \%$ e coeficiente de comportamento de 2,0. Para a capacidade resistente dos solos de fundação consideraram-se $200 \mathrm{kPa}$

Após a quantificação das ações, procedeu-se às verificações da segurança da estabilidade da estrutura. Para o efeito, todas as seções transversais dos elementos estruturais foram dimensionadas de forma a verificar os estados últimos de resistência e os estados-limite de utilização, adotando-se as disposições preconizadas em REBAP (PORTUGAL, 1983).

Os pavimentos dos pisos serão maioritariamente executados com lajes de vigotas protendidas. Esses pavimentos têm um funcionamento estrutural unidirecional, resistindo essencialmente a momentos flectores positivos. O apoio desses pavimentos faz-se diretamente em vigas.

Pelo reduzido esforço axial existente nas vigas, a armadura longitudinal é calculada para a flexão simples e os estribos associados ao esforço transverso. Por sua vez, os pilares foram dimensionados tendo em conta que estão sujeitos à flexão desviada composta e considerando os efeitos de segunda ordem. Para a fundação dos pilares previu-se a execução de sapatas isoladas.

Após o dimensionamento da solução estrutural tradicional adotada para o edifício em estudo obteve-se como resultado a estrutura representada nas Figuras 5 a 7. É possível observar nessas figuras as plantas estruturais do edifício com os diferentes elementos estruturais: sapatas, pilares, vigas e lajes. Para exemplificar a pormenorização dos elementos estruturais de betão armado, apresenta-se a distribuição de armadura num troço de viga, onde é possível observar o comprimento de amarração de cada varão e a distribuição dela na secção transversal da viga (Figura 8). Esse tipo de pormenorização foi também efetuado para os restantes elementos estruturais. Com base nos resultados do dimensionamento realizou-se a quantificação de todos os materiais, que se resume na Tabela 1 (terceira coluna).

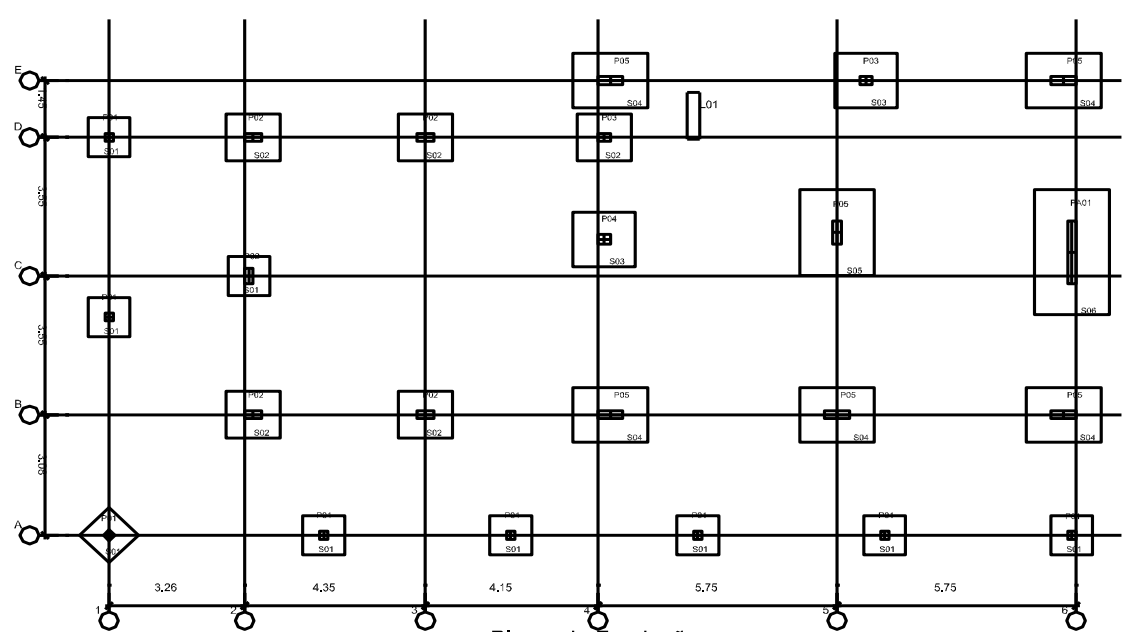

Figura 5 - Planta de fundações - Solução tradicional 


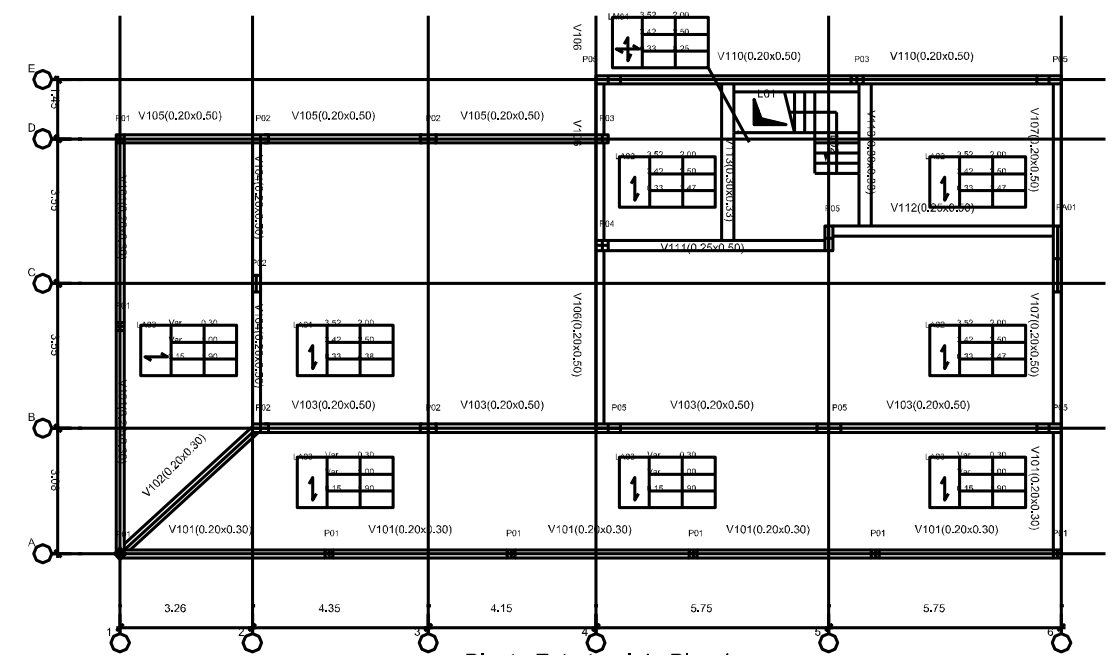

Figura 6 - Planta estrutural do piso 1 - Solução tradicional

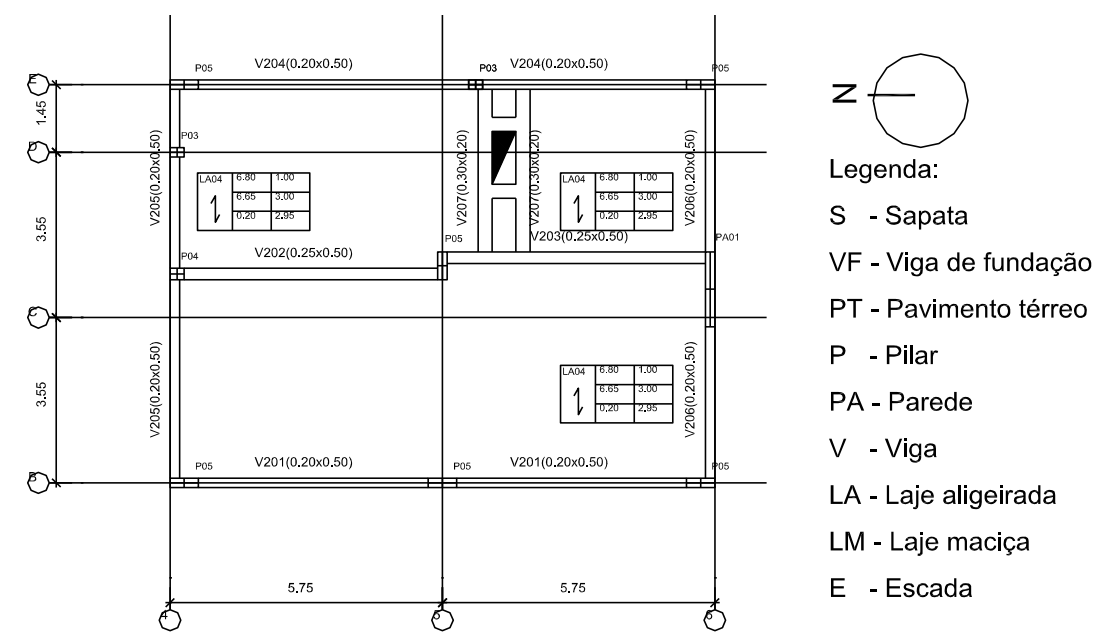

Figura 7 - Planta estrutural da cobertura - Solução tradicional

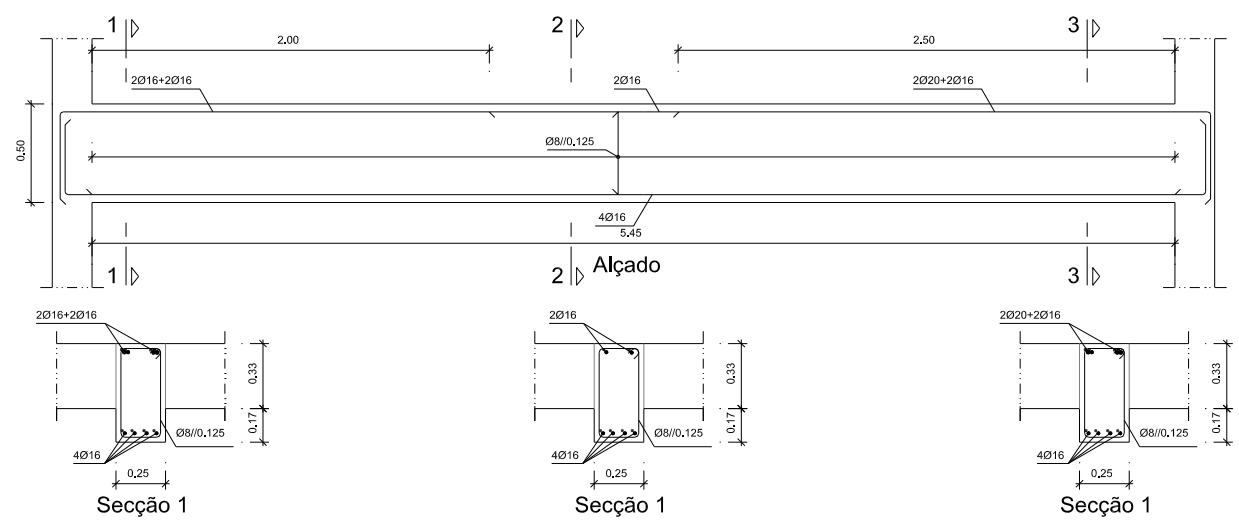

Figura 8 - Pormenores de uma viga - Solução tradicional 


\begin{tabular}{lcrrr}
\hline Material & Unidade & Tradicional & Sustentável I & Sustentável II \\
\hline Betão & $\mathrm{m}^{3}$ & 120,25 & 28,00 & 28,00 \\
Aço & $\mathrm{kg}$ & $6.480,00$ & 455,00 & 455,00 \\
Argamassa & $\mathrm{m}^{3}$ & 24,80 & ------ & ----- \\
Tijolo & un & $12.969,00$ & ----- & ---- \\
Pedra & $\mathrm{m}^{3}$ & 39,00 & 128,60 & 128,60 \\
BTC & un & ------ & $18.000,00$ & $18.000,00$ \\
Argamassa (terra) & $\mathrm{m}^{3}$ & ----- & 48,20 & 48,20 \\
Madeira & $\mathrm{kg}$ & ------ & $16.211,05$ & ------ \\
Madeira (velha) & $\mathrm{kg}$ & ----- & ----- & $18.650,00$ \\
\hline
\end{tabular}

Tabela 1 - Quantidade de material por solução estrutural

\section{Solução sustentável I}

Para a solução estrutural sustentável I, procedeu-se inicialmente ao pré-dimensionamento dos elementos estruturais, considerando as dimensões mínimas admissíveis para todos esses elementos, de acordo com o preconizado no EC6 (EUROPEAN COMMITTEE FOR STANDARDIZATION, 2005a) e EC5 (EUROPEAN COMMITTEE FOR STANDARDIZATION, 2004). Posteriormente, dimensionaram-se todos esses elementos utilizandose folhas de cálculo em Excel especialmente desenvolvidas para o efeito.

As unidades consideradas para as alvenarias resistentes da solução estrutural sustentável I foram os blocos de BTC, classificados segundo o EC6 como uma pedra artificial. Neste estudo não se consideraram os adobes, uma vez que a resistência a compressão $\left(f_{b}\right)$ dos blocos de adobe é inferior à resistência mínima admitida no EC6 (EUROPEAN COMMITTEE FOR STANDARDIZATION, 2005a), que corresponde a 4,00 MPa.

Buson (2009) apresenta no seu trabalho uma análise detalhada de todos os aspectos relacionados com a produção dos BTC, desde a seleção dos componentes, tipos de solos, estabilizantes, processos de fabrico, equipamento utilizado, processo de cura e características finais dos blocos que condicionam o seu desempenho em obra.

Para a obtenção de resistências da ordem de grandeza do valor mínimo enunciado no EC6, segundo Grande (2003), é necessário ajustar os processos e os materiais para o fabrico dos blocos de terra compactada, para se obter melhor homogeneidade, resistência e estabilidade. Também recomenda o uso de uma percentagem de cimento entre $10 \%$ e $12 \%$, a misturar com a terra, e que o período de secagem dos blocos deverá ser aproximadamente de 120 dias. De acordo com os ensaios realizados por Grande (2003), essas recomendações, quando implementadas, podem gerar blocos de terra da classe U4 $\left(\mathrm{f}_{\mathrm{b}}=4,00 \mathrm{MPa}\right)$. Como referido anteriormente, o tipo de bloco considerado neste estudo para as paredes resistentes é o BTC, admitindo-se que a sua resistência a compressão é de 4,0 $\mathrm{MPa}$. Para as juntas de assentamento considerou-se uma argamassa da classe M5 (5,0 MPa), de acordo com o EC6 (EUROPEAN COMMITTEE FOR STANDARDIZATION, 2005a).

Para o pavimento do piso 1, para a cobertura e para as escadas foram consideradas vigas e soalho de madeira de pinho bravo (Pinus pinaster), mais concretamente da classe E, de acordo com o EC5 (EUROPEAN COMMITTEE FOR STANDARDIZATION, 2004).

A fundação adotada para as paredes resistentes de BTC é do tipo sapata corrida (tipo baldrame) em pedra de calcário duro. Optou-se por esse material por ser natural e local.

A análise estrutural desenvolvida para a solução sustentável I estudada seguiu os princípios e os procedimentos referidos anteriormente para a solução tradicional de betão armado. Para o peso específico dos BTC foi considerado o valor de $18,00 \mathrm{kN} / \mathrm{m}^{3}$, e para a madeira, o valor de 5,80 $\mathrm{kN} / \mathrm{m}^{3}$. As restantes ações consideradas foram análogas às definidas na seção anterior para a solução estrutural tradicional.

Para os elementos estruturais de madeira também foi desenvolvida e utilizada uma folha de cálculo em Excel com base no prescrito no EC5 (EUROPEAN COMMITTEE FOR STANDARDIZATION, 2004). $\mathrm{O}$ apoio desses pavimentos faz-se diretamente nas paredes resistentes de BTC.

Por sua vez, as paredes de BTC foram dimensionadas tendo em conta o especificado no RSA (PORTUGAL, 1985) e no EC6 (EUROPEAN COMMITTEE FOR STANDARDIZATION, 2005a), recorrendo também a uma folha de cálculo em Excel desenvolvida para o efeito. Pelo fato de 
não se dispor de um programa de cálculo automático para o dimensionamento de alvenarias e de estruturas de madeira, limitou-se esse dimensionamento a uma análise 2D. Contudo, uma análise estrutural em 3D também foi processada para uma avaliação mais realista, usando-se para o efeito um programa de cálculo automático capaz de efetuar análise por elementos finitos. A estrutura do edifício foi simulada por elementos de barra e de casca. $\mathrm{Na}$ Figura 9 apresenta-se a malha de elementos finitos utilizada para modelar o edifício.

As tensões normais ocorridas nas paredes resistentes de BTC não excederam a capacidade resistente do material, e como tal a estabilidade foi verificada.

Do dimensionamento da solução estrutural sustentável I adotada para o edifício em estudo chegou-se aos resultados expostos nas Figuras 10 a 12 , onde se observa a localização e a distribuição das paredes estruturais e as sapatas/lintéis que as suportam. Nas mesmas figuras representa-se a orientação das vigas de pavimento, sobre as quais se apoia o soalho, bem como a definição das diferentes tipologias de paredes utilizadas, cuja espessura e arranjo dos blocos dependem do nível de carga a que estas estão sujeitas. Representa-se na Figura 13 um pormenor tipo de cada tipologia das paredes adotadas, bem como um pormenor da solução corrente para execução das aberturas nas paredes, com recurso a uma viga/lintel de madeira para suporte dos blocos da parede acima da abertura. Finalmente, apresenta-se ainda o pormenor de apoio do soalho nas vigas de pavimento.

Da comparação entre as plantas estruturais da solução sustentável com a solução tradicional, nota-se que a solução sustentável apresenta uma área de paredes consideravelmente superior à solução tradicional, onde apenas surgem pilares esbeltos, sendo a execução das paredes divisórias nesta solução realizada com tijolos cerâmicos vazados, de espessuras menores. $\mathrm{Na}$ solução sustentável as paredes são estruturais, servindo ao mesmo tempo e delimitação dos espaços e vedação da habitação, enquanto na solução tradicional estas não assumem a função estrutural.

Com os resultados de dimensionamento e pormenorização das estruturas, representadas nas Figuras 10 a 13, foi então possível quantificar os materiais inerentes à construção do edifício com base nessa solução, que se resumem na Tabela 1 .
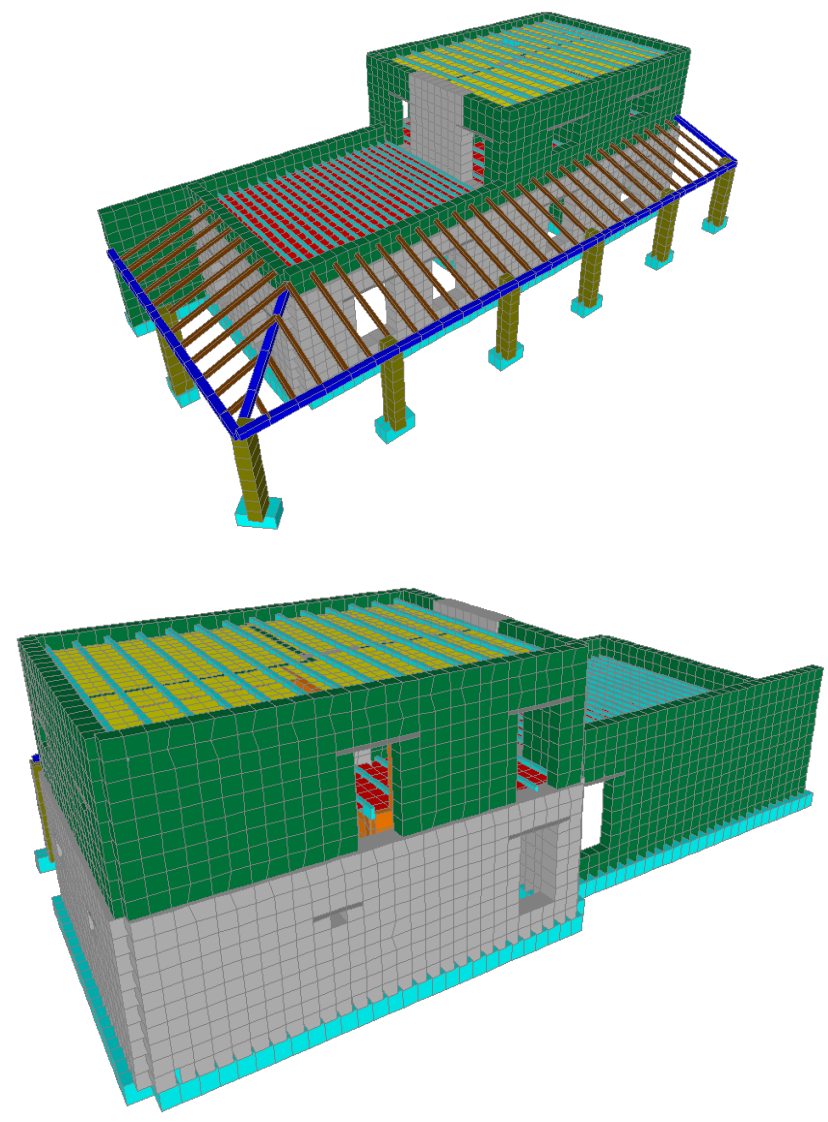

Figura 9 - Malha de elementos finitos do modelo numérico 


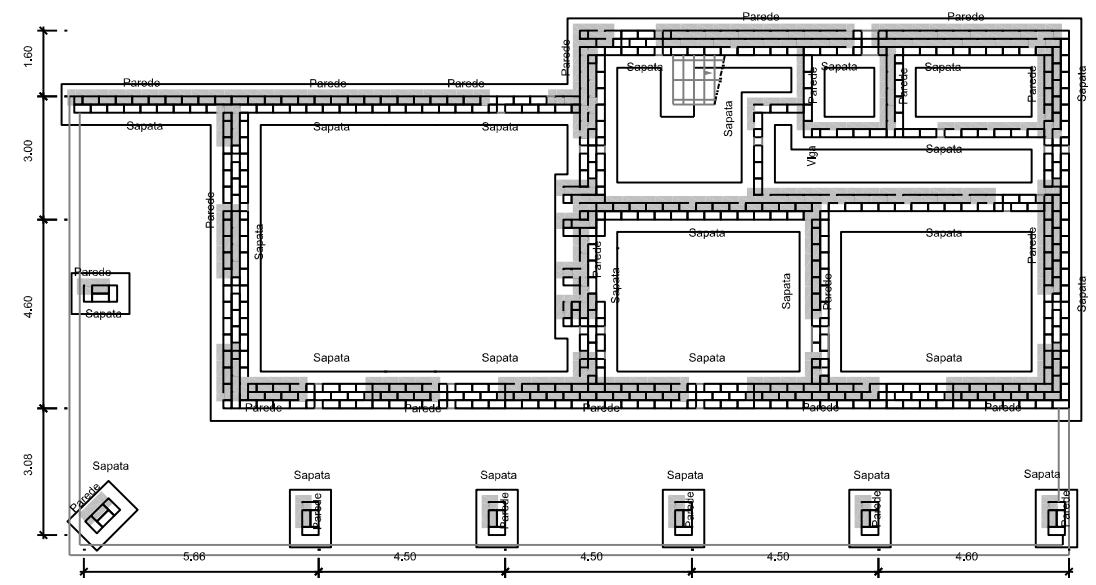

Figura 10 - Planta de fundações - Solução sustentável I

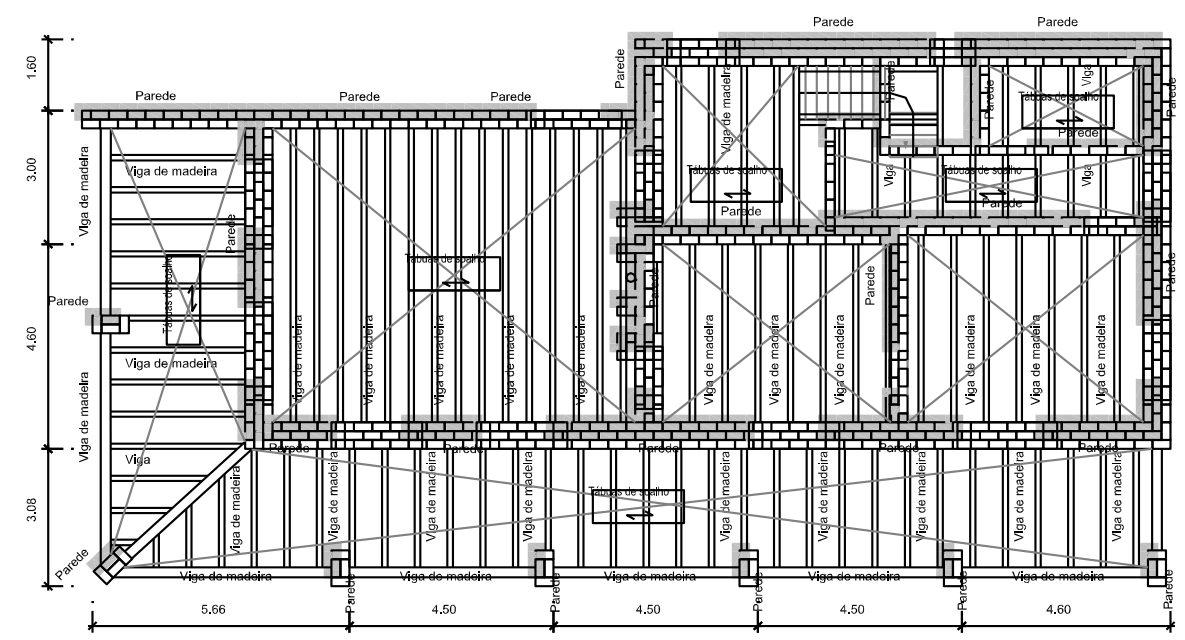

Figura 11 - Planta estrutural do piso 1 - Solução sustentável I

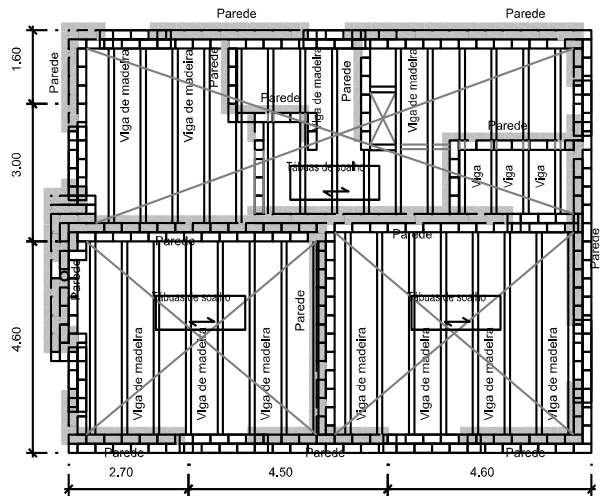

Figura 12 - Planta estrutural da cobertura - Solução sustentável I 

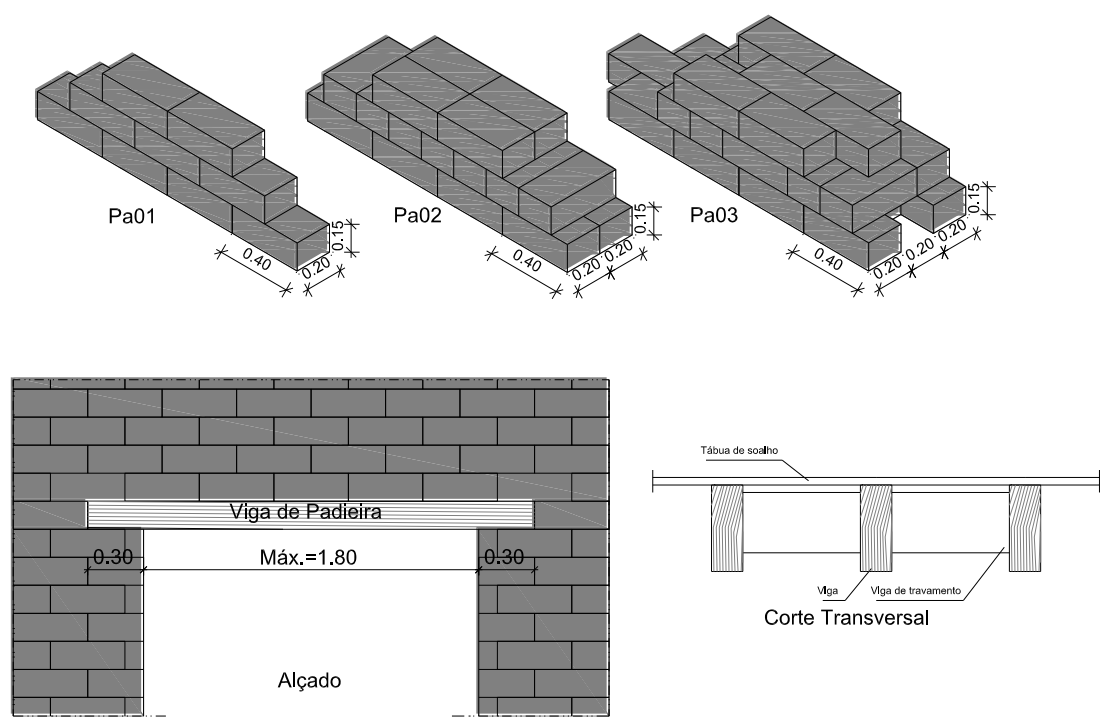

Figura 13 - Pormenores construtivos - Solução sustentável I

\section{Solução sustentável II}

Para a solução estrutural sustentável II, todo o processo de dimensionamento foi semelhante ao usado e descrito para a solução sustentável I, quer em termos de regulamentação, de ações consideradas e de metodologia de cálculo. Os materiais previstos também foram os mesmos, sendo apenas exceção o fato de se ter proposto a reutilização de madeira de pinho bravo (Pinus pinaster) proveniente da demolição de outros edifícios. As plantas estruturais com a localização dos diversos elementos são também semelhantes à solução estrutural sustentável I, bem como os pormenores construtivos. Apenas a dimensão dos elementos de madeira sofrem alterações devido à diferente resistência entre os tipos de madeira utilizada nas duas soluções sustentáveis alternativas.

A análise estrutural desenvolvida para a solução sustentável II seguiu os princípios e os procedimentos referidos anteriormente para a solução sustentável I, tendo apenas em consideração que a madeira a utilizar é proveniente de demolições. Ainda é frequente a ocorrência de processos de demolição integral de edifícios antigos, que geralmente são considerados obsoletos, para permitir a construção de edifícios novos. Simultaneamente, também ainda se verifica que nesses processos continua a não existir uma filosofia de aproveitamento dos materiais construtivos.

Pedras, telhas cerâmicas, vigas de madeira e elementos metálicos são exemplos de materiais construtivos passíveis de serem aproveitados e posteriormente reutilizados. Segundo Ross e Wang (2005), os elementos de madeira tal como vigas provenientes de edifícios antigos podem apresentar patologias que resultem num desperdício de aproveitamento superior a 50\%, embora seja também referido que existem muitos casos em que esse desperdício seja inferior a $10 \%$ (aproveitamento superior a 90\%). As principais patologias estruturais identificadas nesses elementos são a existência de grandes fissuras, a existência de nós e o apodrecimento dos extremos dos elementos (zonas de apoio).

Relativamente à capacidade resistente da madeira reutilizada, Marzo (2006) indica que os elementos de madeira antiga analisados apresentam geralmente diminuição do módulo de elasticidade em cerca de $25 \%$, resultante de vários fatores, tal como envelhecimento do material, excentricidades resultantes de fraca execução, ligações deficientes, entre outros. Por sua vez, Pilt et al. (2009) consideraram a ocorrência de diminuição da capacidade resistente da madeira antiga de cerca de $15 \%$. Este foi o valor adotado neste estudo para estimar a redução da capacidade resistente da madeira a reutilizar, e os resultados estão apresentados nas Figuras 10 a 13. Com esses resultados foi possível quantificar os materiais inerentes à construção do edifício com base nessa solução (Tabela 1). 


\section{Metodologia para o cálculo do consumo energético e de emissão de $\mathrm{CO}_{2}$}

A qualquer material de construção está sempre associado um consumo energético e um teor de emissão de gases poluentes para a atmosfera resultante da extração da matéria-prima, do seu transporte, da sua eventual transformação noutro material, entre outras tarefas. É possível estimar o consumo desses parâmetros ambientais associado a cada uma das tarefas e, consequentemente, de todo o ciclo de vida útil de dado material.

A análise de diversos estudos nessa temática, por exemplo, Kanghee et al. (2007), Centre for Environmental Studies (2001) e Baird, Alcorn e Haslam (1997), evidencia alguma discrepância nos valores propostos para esses parâmetros. Segundo Baird, Alcorn e Haslam (1997), essas discrepâncias podem estar associadas a diferentes aspectos, associados à definição de diferentes ciclos de vida nas análises, a períodos diferentes, a diferentes sistemas de fabrico, entre outras.

Segundo Torgal e Jalali (2007), a aplicação generalizada de análises de ciclos de vida no setor da construção, e no caso particular dos materiais usados na construção, pressupõe antes de tudo a existência de levantamentos exaustivos sobre os impactos ambientais desses materiais ao longo da sua vida útil, algo que dificilmente pode ser extrapolado a partir de estudos realizados noutros países, devido a diferenças óbvias que se prendem com diferentes contextos tecnológicos e económicos. Para Kanghee et al. (2007), um dos principais fatores das divergências nessas análises comparativas poderá até residir na metodologia utilizada. Estes autores afirmam que um método de estimativa indireta dos parâmetros de dado material poderá ser tão eficaz como uma análise do ciclo de vida do mesmo material.

$\mathrm{Na}$ Tabela 2 apresentam-se alguns valores de referência do gasto energético de diversos materiais de construção propostos por diferentes autores. Neste trabalho foi adotada a metodologia proposta por Kanghee et al. (2007), em que se comparou a determinação dos parâmetros de gasto energético e de emissão de $\mathrm{CO}_{2}$ relacionados com a construção de edifícios e que serviu de base para a obtenção dos valores unitários de gastos energéticos e de emissões de $\mathrm{CO}_{2}$ adotados para os diferentes materiais de construção abordados neste trabalho de investigação (Tabela 3).

\begin{tabular}{l|c|c|c|c}
\hline Material & Kanghee $^{\mathbf{1}}$ & Leiden $^{\mathbf{2}}$ & Baird $^{\mathbf{3}}$ & Alcorn $^{\mathbf{3}}$ \\
\hline Betão & 0,52 & 0,48 & 1,99 & 1,95 \\
\hline Aço & 38,66 & ----- & 59,00 & 8,90 \\
\hline Argamassa & 0,40 & 0,88 & 2,49 & 1,33 \\
\hline Tijolo & 2,95 & 0,15 & 2,50 & ----- \\
\hline BTC & 0,06 & ----- & 0,42 & ----- \\
\hline Pedra & 0,15 & ----- & 0,30 & 0,10 \\
\hline Madeira & 4,44 & ----- & 8,08 & 1,10 \\
\hline Areia & 0,05 & ----- & 0,04 & 0,10 \\
\hline Cimento & 3,33 & ----- & 8,98 & 7,80 \\
\hline Terra & 0,05 & ----- & ----- & ----- \\
\hline
\end{tabular}

Fonte: ${ }^{1}$ Kanghee et al. (2007), ${ }^{2}$ Centre For Environmental Studies (2001), ${ }^{3}$ Baird, Alcorn e Haslam (1997).

Tabela 2 - Gasto energético (MJ/kg) 


\begin{tabular}{|c|c|c|c|c|}
\hline \multirow{2}{*}{ Material } & \multirow{2}{*}{ Unidade } & Custo & Consumo de energia & Emissão $\mathrm{CO}_{2}$ \\
\hline & & (€/Unidade) & (MJ/Unidade) & $\left(\mathrm{CO}_{2} /\right.$ Unidade $)$ \\
\hline Betão & $\mathrm{m}^{3}$ & 50,25 & 1292,24 & 99,43 \\
\hline Aço & $\mathrm{kg}$ & 0,63 & 38,66 & 3,72 \\
\hline Argamassa & $\mathrm{m}^{3}$ & 50,45 & 961,40 & 63,91 \\
\hline Tijolo & un & 0,31 & 14,78 & 1,11 \\
\hline Pedra & $\mathrm{m}^{3}$ & 15,00 & 365,26 & 26,90 \\
\hline BTC & un & 0,15 & 1,45 & 0,09 \\
\hline Argamassa (terra) & $\mathrm{m}^{3}$ & 48,88 & 931,48 & 61,92 \\
\hline Madeira & $\mathrm{kg}$ & 0,40 & 4,44 & 0,38 \\
\hline Madeira (velha) & $\mathrm{kg}$ & 0,002 & 0,05 & 0,0035 \\
\hline
\end{tabular}

Tabela 3 - Parâmetros dos materiais

\section{Resultados e discussão}

Para a obtenção dos parâmetros de custo, de gasto energético e de emissão de $\mathrm{CO}_{2}$ para a madeira antiga (solução sustentável II) considerou-se que o custo dessa madeira é de apenas $20 \%$ do respectivo valor adotado para a madeira nova. Por sua vez, o gasto energético e a emissão de $\mathrm{CO}_{2}$ para esse tipo de material foram considerados referentes apenas às tarefas de desmonte e de transporte até ao local da obra. Nesse contexto, considerou-se que o material seria proveniente de demolições vizinhas e de localizadas a uma distância máxima de $5 \mathrm{~km}$.

Neste estudo, os gases poluentes estão convertidos em valores equivalentes de $\mathrm{CO}_{2}$, e como unidade funcional considerou-se a área total bruta de construção, que é igual a $391,5 \mathrm{~m}^{2}$.

Os valores de gasto energético e de teor de emissão de $\mathrm{CO}_{2}$ para a atmosfera apresentam-se nas Tabelas 2 e 4, para as três soluções estruturais estudadas para a construção de habitação unifamiliar considerada neste estudo.

Para a obtenção dos valores dos parâmetros por metro quadrado considerou-se para a solução estrutural tradicional uma área de construção total bruta de $362,51 \mathrm{~m}^{2}$, e para as restantes soluções, uma área de $398 \mathrm{~m}^{2}$.

Neste estudo de caso, estima-se que a opção de solução estrutural sustentável I permitirá alcançar uma redução do custo de material em cerca de $13,60 \%$, do gasto energético de $65,10 \%$, e uma redução de emissão de $\mathrm{CO}_{2}$ de 67,90\% (Tabela 4).
Contudo, a opção de solução estrutural sustentável II revelar-se-á mais económica e muito mais amiga do ambiente porque permitirá alcançar uma redução do custo de material em cerca de 45,20\%, do gasto energético de $75,30 \%$, e uma redução de emissão de $\mathrm{CO}_{2}$ de 78,40\% (Tabela 4).

Esses resultados demonstram que a opção pela solução estrutural sustentável II é de fato ambientalmente bastante mais sustentável.

Com a definição de todos os parâmetros de comparação das diferentes soluções estruturais é possível associar a cada um deles um consumo energético equivalente - neste caso foi considerado o kWh.

Para tal, no contexto português, correntemente e segundo os valores de mercado, $1 \mathrm{kWh}$ de energia equivale a 0,12 euro, $€$, (dados fornecidos pela empresa EDP - Energias de Portugal), uma unidade de gasto energético (MJ) é equivalente a 0,28 $\mathrm{kWh}$, e a cotação de uma tonelada de $\mathrm{CO}_{2}$ é estimada em cerca de $14 €$ (Público, 2010/01/23). Com todos esses parâmetros pode-se então obter o consumo energético equivalente inerente a cada solução estrutural estudada em função da unidade de energia, o $\mathrm{kWh}$.

Na Tabela 5 apresentam-se os valores globais totais dos parâmetros ambientais usados na comparação das três soluções estruturais (tradicional, sustentável I e sustentável II) traduzidos em consumo energético $(\mathrm{kWh})$. 


\begin{tabular}{l|c|c|c|c|c}
\hline Parâmetro & Unidade & Tradicional & Sustentável I & Sustentável II & Melhor solução \\
\hline Custo & $€ / \mathrm{m}^{2}$ & 44,09 & 38,10 & 24,15 & Sustentável II \\
\hline Gasto energético & $\mathrm{MJ} / \mathrm{m}^{2}$ & 1753,60 & 612,36 & 433,86 & Sustentável II \\
\hline Emissão de $\mathrm{CO}_{2}$ & $\mathrm{~kg}-\mathrm{CO}_{2} / \mathrm{m}^{2}$ & 146,46 & 46,99 & 31,67 & Sustentável II \\
\hline
\end{tabular}

Tabela 4 - Comparação das soluções estruturais por parâmetro

\begin{tabular}{l|c|c|c|c|c}
\hline Parâmetro & Unidade & Tradicional & Sustentável I & Sustentável II & Melhor solução \\
\hline Custo & MWh & 133,17 & 126,35 & 80,09 & Sustentável II \\
\hline Gasto energético & MWh & 177,98 & 68,24 & 48,34 & Sustentável II \\
\hline Emissão de $\mathrm{CO}_{2}$ & MWh & 6,19 & 2,18 & 1,47 & Sustentável II \\
\hline
\end{tabular}

Tabela 5 - Comparação das soluções estruturais em termos de consumo energético equivalente

A análise desses resultados permite constatar que a solução estrutural sustentável II apresenta valores dos três parâmetros (custo de material, gasto energético e teor de emissão de $\mathrm{CO}_{2}$ para a atmosfera) significativamente inferiores em relação aos respectivos valores obtidos para as soluções estruturais tradicional e sustentável I.

A solução sustentável I apresenta relativamente à solução tradicional uma diminuição de $5,12 \%$ no custo dos materiais, uma diminuição de $61,66 \%$ do gasto energético e uma diminuição de $64,78 \%$ nas emissões de $\mathrm{CO}_{2}$. A solução sustentável II apresenta uma diminuição de $39,85 \%$ no custo dos materiais, uma diminuição de $72,83 \%$ do gasto energético e uma diminuição nas emissões de $\mathrm{CO}_{2}$ de $76,25 \%$

Mateus e Bragança (2004) referem que em Portugal se estima que os edifícios (habitação e serviços) durante a fase de utilização são responsáveis pelo consumo de cerca de $20 \%$ dos recursos energéticos nacionais, por $6,7 \%$ do consumo de água e pela produção anual de 420 milhões de metros cúbicos de águas residuais. De acordo com os resultados obtidos neste estudo, a adoção de uma solução construtiva recorrendo a materiais naturais poderá conduzir a uma diminuição de $65 \%$ do gasto energético inerente aos materiais adotados na construção. No entanto, refere-se que esse tipo de soluções mais sustentáveis também contribui de forma significativa para a diminuição da necessidade de recursos energéticos durante a utilização da habitação.

Tal como referem Mateus e Bragança (2004), “[...] o desenvolvimento e a aplicação de metodologias de avaliação da sustentabilidade são aspectos fundamentais nas diversas tomadas de decisão que procurem a criação de edifícios mais sustentáveis.".

Por sua vez, na Tabela 6 é apresentado o período, em anos, em que é possível amortizar o custo dos materiais de construção, caso se pretenda optar por uma das soluções mais sustentáveis propostas, em detrimento da solução tradicional, e que para efeitos de comparação se considera o consumo energético médio anual de uma habitação unifamiliar portuguesa. De acordo com os dados fornecidos pela EDP, esse valor é de 3.346 $\mathrm{kWh} / \mathrm{ano}$.

Para efeitos de comparação, foi tomado como base o valor do custo energético da solução tradicional e por ser a solução menos económica (Tabela 5). 


\begin{tabular}{l|c|c|c|c|c}
\hline Parâmetro & Unidade & Tradicional & Sustentável I & Sustentável II & Melhor solução \\
\hline Custo & anos & ---- & 2,04 & 15,87 & Sustentável II \\
\hline Gasto energético & anos & ---- & 32,80 & 38,75 & Sustentável II \\
\hline Emissão de $\mathrm{CO}_{2}$ & anos & ---- & 1,20 & 1,41 & Sustentável II \\
\hline Total & anos & ----- & 36,04 & 56,02 & Sustentável II \\
\hline
\end{tabular}

Tabela 6 - Comparação das soluções estruturais em função do consumo energético médio anual

Para o dono de obra, a vantagem direta está associada ao custo da construção (primeira linha da Tabela 6). Ele se beneficiará de 2 anos ou de 16 anos de consumo energético médio de utilização de uma habitação se optar pela solução estrutural sustentável I ou II respectivamente. Por sua vez, essa poupança poderá ser investida na melhoria do conforto da habitação, nomeadamente no aquecimento, tendo em conta que a fatura a pagar por esse serviço em Portugal é bastante elevada e excede o orçamento de muitas famílias.

Em termos do país (Portugal) e nos contextos do Protocolo de Quioto (UNITED NATIONS, 1998) mais concretamente nas alíneas i), iv) e vi) do artigo 2.1.a, as soluções estruturais mais sustentáveis propostas poderão dar um contributo significativo porque permitirão simultaneamente reduzir a emissão de gases poluentes para a atmosfera e o consumo de energia elétrica proveniente de energias não renováveis. De acordo com os resultados expostos na Tabela 6 (última coluna), as soluções sustentáveis I e II permitirão alcançar um ganho de consumo energético médio anual de 36 anos e de 56 anos respectivamente. Essa informação é muito importante, tendo em conta que o período de vida útil considerado para uma estrutura é de 50 anos.

De forma a estimular os cidadãos a optar por soluções construtivas mais sustentáveis, o Estado deveria criar mecanismos de incentivos e de apoio como benefícios fiscais.

Neste estudo os valores de sequestro de carbono para a madeira não foram tidos em conta. Se esses valores tivessem sido considerados, então os benefícios económicos e ambientais inerentes às soluções estruturais mais sustentáveis propostas seriam ainda mais expressivos.

\section{Conclusões e considerações finais}

Durante a realização deste trabalho de investigação constatou-se que ainda existe uma grande escassez de informação e de regulamentação relativa à aplicação de materiais naturais e, em particular, à utilização do BTC.

Três soluções estruturais para uma habitação típica unifamiliar portuguesa foram definidas, dimensionadas e comparadas em termos de custo e ambientais.

Foram propostos diversos pormenores construtivos para a execução das diferentes soluções estruturais propostas. Os pormenores construtivos propostos para as soluções mais sustentáveis têm uma importância acrescida, dado que ainda existe uma falta de experiência técnica para a execução desse tipo de construções.

Diversos autores propõem valores unitários de gasto energético e de teor de emissão de gases poluentes para a atmosfera para materiais de construção que são divergentes. Pensa-se que a causa dessa divergência esteja relacionada com o fato de serem considerados períodos temporais de vida do material distintos em cada estudo. Neste trabalho, o período temporal adotado está compreendido entre a obtenção da matéria-prima e a aplicação do material em obra. Caso fosse adotado o ciclo de vida completo do material como período temporal de análise, seria necessário ainda contemplar as fases de manutenção, conservação, demolição e reciclagem.

A unidade funcional adotada na quantificação dos parâmetros ambientais de gasto energético e de teor de emissão de gases poluentes para a atmosfera foi a área bruta de construção. O teor de emissão de gases poluentes para a atmosfera está convertido em teor equivalente de emissão de $\mathrm{CO}_{2}$ para a atmosfera.

A comparação entre os valores obtidos para os três parâmetros em cada solução estrutural estudada permitiu concluir que a solução estrutural mais sustentável que contempla paredes resistentes de BTC, fundações de pedra natural e local, pavimentos, cobertura e escadas de madeira antiga reutilizada é claramente a mais vantajosa em termos económicos e ambientais, havendo uma redução de custos da ordem dos $40 \%$ e de gasto energético e de emissão de $\mathrm{CO}_{2}$ superior a $75 \%$. 
$\mathrm{O}$ fato de se reutilizar madeira antiga possibilita a diminuição do número de árvores a abater e consequentemente um aumento do sequestro de carbono.

O aproveitamento de materiais de construção, tais como pedra, telhas cerâmicas, elementos de madeira e metálicos, permitirá a diminuição da quantidade de detritos resultantes da demolição de edifícios.

Esses resultados podem contribuir para estimular a aplicação desse tipo de soluções estruturais mais sustentáveis em edifícios de habitação unifamiliar e em alguns edifícios de habitação coletiva ou outro tipo de construção. Os ganhos de consumo energético médio anual associados a esse tipo de solução construtiva são muito relevantes e, por isso, o Estado deveria criar mecanismos de incentivo. Na perspectiva do dono de obra, o benefício económico resultante poderá ser direcionado para a melhoria do conforto da habitação.

Comprovam-se, assim, a viabilidade e o benefício da utilização de materiais naturais em edifícios unifamiliares. É possível reduzir o custo de construção quando são utilizados materiais naturais, além dos enormes benefícios ambientais. Este estudo constitui-se, portanto, como uma contribuição metodológica na aplicação de materiais naturais no setor da construção de edifícios de habitação unifamiliar, quantificando o efeito dessas soluções em termos de custo, consumos energéticos e emissões de $\mathrm{CO}_{2}$.

\section{Referências}

AUROVILLE EARTH INSTITUTE. Welcome to Earth Architecture! Auroshilpam, 2010.

Disponível em: <http://www.earth-auroville.com>. Acesso em: 18 fev. 2010.

BAIRD, G.; ALCORN, A.; HASLAM, P. The Energy Embodied in Building Materials. New Zealand: IPENZ Transactions, 1997.

\section{BUSON, M. A. KRAFTTERRA:}

desenvolvimento e análise preliminar do desempenho técnico de componentes de terra com a incorporação de fibras de papel kraft provenientes da reciclagem de sacos de cimento para vedação vertical. 2009. 135 f. Tese (Doutorado em Arquitetura e Urbanismo) Faculdade de Arquitetura e Urbanismo, Universidade de Brasília, Brasília, DF, 2009.
CENTRE FOR ENVIRONMENTAL STUDIES

CML 2 Baseline Method (2000). Leiden:

University of Leiden, 2001. Disponível em: <http://www.pre.nl/simapro/impact_assessment_m ethods.htm\#CML2>. Acesso em: 18 fev. 2010.

CORTÉS, M. Inovación Tecnológica de Construcción en Tierra, Posibilidades en la Arquitectura Contemporânea. In: SEMINARIO IBEROAMERICANO DE CONSTRUCCIÓN CON TIERRA, 8., 2009, Tucumán. Anais... Tucumán: CRIATIC-FAU-UNT, 2009.

\section{EUROPEAN COMMITTEE FOR}

STANDARDIZATION. Eurocode 5: design of timber structures: ENV 1995-1. Brussels, 2004.

\section{EUROPEAN COMMITTEE FOR}

STANDARDIZATION. Eurocode 6: design of masonry structures: ENV 1996-1. Brussels, 2005a.

\section{EUROPEAN COMMITTEE FOR}

STANDARDIZATION. Eurocode 8: design of masonry structures: ENV 1998-1. Brussels, 2005a.

GRANDE, F. M. Fabricação de Tijolos

Modulares de Solo-Cimento por Prensagem Manual com e sem Adição de Sílica Activa. 2003. 165 f. Dissertação (Mestrado em Arquitetura) - Escola de Engenharia de São Carlos, Universidade de São Paulo, São Carlos, 2003.

KANGHEE, L. et al. The Estimation of the Functional Unit on Energy Consumption and $\mathrm{CO}_{2}$ Emission Concerned with Construction of Building. In: INTERNATIONAL CONFERENCE ON SUSTAINABLE BUILDING ASIA, 2007, Seoul. Anais... Seoul: SB07, 2007.

MARZO, A. Methodology for the Analysis of Complex Historical Wooden Structures. Pollack Periodica, Budapest, v. 1, n. 1, p. 35-52, abr. 2006.

\section{MATEUS, R.; BRAGANÇA, L. Avaliação da Sustentabilidade da Construção: \\ desenvolvimento de uma metodologia para a avaliação da sustentabilidade de soluções construtivas. Guimarães: Universidade do Minho, 2004. Disponível em: <http://www.repositorium.sdum.uminho.pt>. Acesso em: 18 fev. 2010.}

MINKE, G. Manual de Construcción en Tierra. Montevideo: Fin de Siglo, 2005. 
PILT, K. et al. Diagnosis of Timber Structures and Archaeological Wood of Cultural Heritage. [S.l.]: WoodCultHer, 2009. Disponível em: <http://www.woodculther.com/wpcontent/uploads/2009/09/Pilt.pdf >. Acesso em: 18 fev. 2010.

PINTO, J. et al. Construção em Tabique na Região de Trás-os-Montes e Alto Douro. In: INTERNATIONAL CONFERENCE ON STRUCTURAL DEFECTS AND REPAIR, 4., 2008, Aveiro. Anais... Aveiro: Civil Engineering Department, University of Aveiro, 2008.

PORTUGAL. Instituto Nacional de Estatística. Mais de 70\% dos Alojamentos Clássicos Ocupados como Residência Habitual, Situam-se na Região de Lisboa e Vale do Tejo e Região Norte. Lisboa, 1998.

PORTUGAL. Ministério da Habitação, Obras Públicas e Transportes. Regulamento de Estruturas de Betão Armado e Pré-Esforçado (REBAP): D.L. 349-C/83. Lisboa, 1983.

PORTUGAL. Ministério da Habitação, Obras Públicas e Transportes. Regulamento de
Segurança e Acções para Estruturas de Edifícios e Pontes (RSA): D.L. 235/85. Lisboa, 1985.

ROOS, R. J.; WANG, X. Condition Assessment of Timbers. Structure Magazine, London, v. 1, n. 9 , p. 32-34, Sept. 2005.

TORGAL, F. P.; JALALI, S, Construção

Sustentável: o caso dos materiais de construção. In: CONGRESSO CONSTRUÇÃO, 3., 2007,

Coimbra. Anais... Coimbra: Universidade de Coimbra, 2007.

UNITED NATIONS. Kyoto Protocol to the United Nations Framework Convention on Climate Change. Kyoto, 1998. 